\title{
Intérêt de l'ozone dans le traitement des maladies parodontales et péri-implantaires
}

\section{RÉSUMÉ}

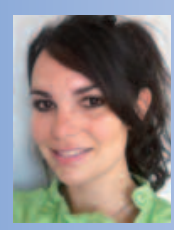

Julie DUCLOT

Chirurgien-dentiste.

Maxime DELVALLE

Ancien interne,

Chirurgien-dentiste

DU d'implantologie clinique de Montpellier.

\section{Manon SERRE}

Ancien interne,

Chirurgien-dentiste.

Philippe GIBERT

PU-PH,

Chef du Service d'Odontologie,

UFR d'Odontologie de Montpellier,

545 avenue du Pr Jean-Louis Viala,

34295 Montpellier Cedex 5.
Les maladies parodontales et péri-implantaires présentent de nombreuses similitudes, en particulier leur origine bactérienne, caractérisée par des bactéries spécifiques. Après les avoir définies, nous aborderons dans une première partie la bactériologie de ces pathologies ainsi que leur mécanisme pathogénique. La deuxième partie de ce travail consistera à faire le point sur les thérapeutiques antiinfectieuses actuelles. Enfin, nous tenterons de préciser l'intérêt de l'utilisation de l'ozone dans le traitement de ces pathologies à travers une étude clinique préliminaire, l'ozone étant un gaz reconnu et utilisé en médecine depuis le début du Xxe siècle, pour ses propriétés bactéricides. 


\section{Introduction}

$>$

Les maladies parodontales et périimplantaires présentent de nombreuses similitudes. Ce sont des états pathologiques inflammatoires d'origine infectieuse. Dans leurs études, Brandes et al. [3] et Lang et al. [8] ont noté que les lésions péri-implantaires et parodontales progressaient de la même manière pendant leur période d'observation. Cependant, la quantité de tissu détruit semble être plus importante autour des implants qu'autour des dents.

Des études [6] ont démontré que le type de flore associé aux maladies parodontales et périimplantaires est riche en gram négatif. Ces études ont montré une forte prévalence de bactéries du complexe rouge (Porphyromonas gingivalis, Treponema denticola et Tannerella forsythia) et du complexe orange (Fusobacterium sp. et Prevotella intermedia), décrits par Socransky et al. [16]. Cependant, dans un certain nombre d'études [6], il a été constaté que Staphylococcus aureus (gram positif) joue un rôle également important dans la pathogénie de nombreux cas de péri-implantite. En effet, des études in vitro ont démontré que $S$. aureus présente une affinité pour les surfaces en titane [5].

Le facteur de surcharge occlusale constitue également une des étiologies de ces deux maladies [17].

Les facteurs de risques pour les parodontites tels que l'hygiène défaillante, la consommation de tabac ou la présence d'un diabète sucré, sont les mêmes que pour les péri-implantites. Il est à noter également que les patients qui ont une susceptibilité pour les parodontites seront plus sensibles aux péri-implantites que les patients n'ayant pas de problèmes parodontaux $[2,13]$.
La parodontite et la péri-implantite étant des infections opportunistes, leur traitement reste le même et sera anti-infectieux [6].

Actuellement, il existe plusieurs types de thérapeutiques anti-infectieuses $[11,14]$ :

- la décontamination/détoxification de la surface implantaire ou radiculaire (méthode mécanique par curetage/surfaçage) ;

- l'antibiothérapie ;

- les traitements antiseptiques.

Pour les maladies implantaires, on peut y ajouter la technique de régénération osseuse guidée (ROG) [14].

Il est à noter que, dans la pratique quotidienne, le traitement de la péri-implantite est plus délicat car l'assainissement de l'environnement de I'implant ne doit pas porter atteinte à sa structure [9]. En effet, le débridement mécanique par I'utilisation de curettes est rendu difficile, de par une complexité d'accès au site chirurgical et de par la nécessité de ne pas dégrader l'état de surface de l'implant.

De ce fait, le principe d'utiliser un gaz, en l'occurrence l'ozone, apparaît comme une piste thérapeutique anti-infectieuse intéressante.

\section{Historique et intérêt de l'ozone}

À la suite des travaux de Fisch et de Dechaume dans les années 1930, I'ozonothérapie a fait son apparition en stomatologie [18]. Les propriétés physicochimiques de ce gaz vont en effet permettre d'obtenir des résultats favorables dans les cas d'alvéolites, de pyorrhées, dans les troubles oculaires d'origine dentaire et dans certaines névralgies; l'ozone atténuait non seulement les 


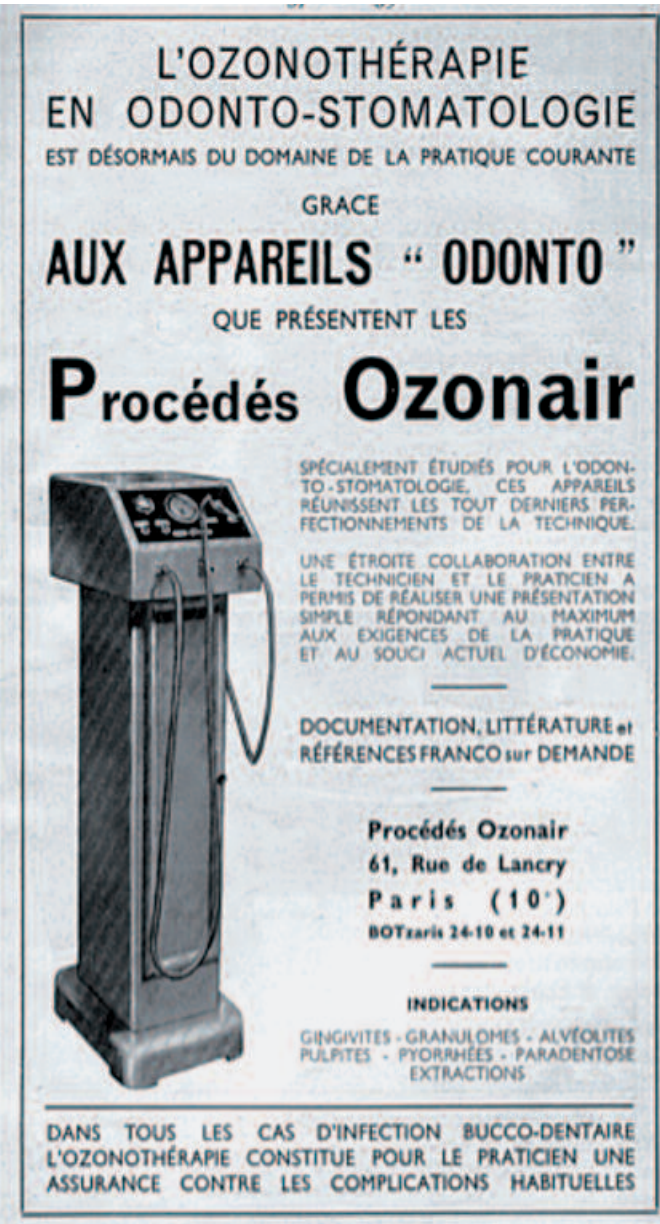

Fig. 1 Source : Société française de l'histoire de l'art dentaire.

Site internet :

http://www.bium.univ-paris5.fr/sfhad/hist_1930-1940.htm. douleurs péridentaires et les inflammations du parodonte mais avait également la propriété de blanchir les dents (et le tartre) (fig. 1).

Une étude de Nagayoshi et al. [12] a démontré in vitro que l'utilisation d'eau ozonisée sur des micro-organismes présents dans la plaque dentaire permet de réduire considérablement la quantité de bactéries. Ces résultats suggèrent que I'eau ozonisée pourrait être utile pour réduire les infections causées par les bactéries contenues dans la plaque dentaire.

Une autre étude [7] a également comparé les effets de l'ozone avec d'autres antiseptiques connus (chlorhexidine, péroxyde d'hydrogène, $\mathrm{CIONa)}$ sur des cellules orales. Cette étude a constaté des effets toxiques sur les deux types de cellules testées. L'ozone a un pouvoir antiseptique. Les résultats ont montré également que l'ozone peut être aisément associé aux autres antiseptiques.

Enfin, des études [1] ont aussi démontré des preuves de biocompatibilité in vitro entre l'ozone et les cellules épithéliales orales et les fibroblastes.

Nous nous sommes donc intéressés à son utilisation dans le traitement des maladies parodontales et des péri-implantites.

\section{Études cliniques préliminaires}

\section{Objectif du projet}

Le but de cette étude consiste à évaluer le rôle bactéricide de l'ozone sur les bactéries parodontopathogènes ainsi qu'à définir son utilisation possible dans le traitement de la maladie parodontale. Pour ce faire, deux protocoles consécutifs ont été mis en place dans le service d'odontologie du CHRU de Montpellier.

\section{Matériel et méthodes}

\section{> Matériel}

Utilisation de l'appareil générateur d'ozone Ozotop qui est muni d'une pièce à main sur laquelle se place l'insert PerioTip, insert capillaire fin spécifiquement employé pour les poches parodontales. 
$>$ Méthodes

Définitions des critères employés :

- Critères d'inclusion :

- patient atteint de parodontite chronique non traitée.

- Critères d'exclusion :

- patient atteint de parodontite agressive ;

- patient traité par antibiothérapie dans les trois mois précédant l'étude ;

- mauvaise hygiène buccodentaire (présence de tartre) :

- utilisation de chlorexidine.

Pendant toute la durée de l'étude, les patients ne devront rien changer à leurs habitudes d'hygiène (brossage classique, non-utilisation de bain de bouche et de dentifrice antiseptiques).
Le principe de l'étude est d'utiliser le générateur d'ozone dans le but d'aider à traiter les maladies parodontales. Pour évaluer son éventuel effet bactéricide, des prélèvements par sondes ADN (pério-analyse) sont réalisés sur les sites concernés.

Les sites de prélèvements sont réalisés sur des poches d'au moins $4 \mathrm{~mm}$ (il s'agit de prélèvements multi-sites dans un tube). Ces sites sont exempts de tartre. Nous avons choisi pour chaque patient plusieurs sites de prélèvement. Sur chaque site, sont évalués l'indice d'hygiène et l'indice de saignement, accompagnés d'un sondage parodontal.

L'injection de l'ozone est de 24 secondes (selon les recommandations du fabricant) dans chaque poche parodontale concernée à l'aide de l'insert PerioTip.

\section{Étude de l'efficacité immédiate}

Ce protocole comprend trois sujets.

Il s'effectue en deux jours :

- à J0, nous avons tout d'abord réalisé des prélèvements bactériens témoins par sondes ADN sur des poches parodontales bien définies. Puis une injection d'ozone au niveau de ces mêmes poches a été effectuée ;

- à J1, des prélèvements bactériens toujours sur ces mêmes sites sont à nouveau effectués afin de mesurer la quantité de germes présente après injection d'ozone.

\section{Résultats (fig. 2)}

\section{$>$ Patient $A$}

Flore totale : J0 : 9,15.109 ;

$$
\text { J1 : } 2,48.10^{9} \text {. }
$$

Les principales bactéries retrouvées chez ce patient sont :

- Eikenella corrodens: sa proportion par rapport à la flore totale est de $2,02 \%$ à J0. À J1, elle sera de $0,55 \%$;

- Fusobacterium nucleatum (Fn) : à J0, on retrouve une proportion de $1,89 \%$ contre $1,65 \%$ à $\mathrm{J} 1$;

- Tannerella forsythensis : à J0, sa proportion est de $1,20 \%$. À J1, on en retrouvera $1,03 \%$;

- Peptostreptococcus micros (Pm) : sa proportion a diminué de $0,26 \%$ à $J 0$ à $0,10 \%$ à J1 ;

- Campylobacter rectus ( $\mathrm{Cr}$ ): seul germe dont la proportion a légèrement augmenté entre J0 et J1 passant de $0,71 \%$ à $0,77 \%$. 


\section{$>$ Patient B}

Flore totale : $\mathrm{J0}: 6,30.10^{9}$;

$$
\text { J1: } 5,24.10^{9} \text {. }
$$

Les principales bactéries retrouvées chez ce patient sont :

- Porphyromonas gingivalis $(\mathrm{Pg})$ : dont la proportion a fortement diminué entre J0 et J1, passant de $3,36 \%$ à $0,63 \%$;

- Fusobacterium nucleatum : ici, également, la quantité de germe a beaucoup baissé : à J0, sa proportion est de $2,94 \%$ contre $0,17 \%$ à J1 ;

- Tannerella forsythensis : sa proportion par rapport à la flore totale est de $0,49 \%$ à $J 0$. À $\mathrm{J} 1$, elle sera de $0,40 \%$;

- Peptostreptococcus micros : sa proportion a diminué de $0,15 \%$ à $\mathrm{J} 0$ à $0,07 \%$ à $\mathrm{J} 1$.

\section{$>$ Patient C}

Flore totale : $\mathrm{J0}: 7,43.10^{9}$;

$$
\mathrm{J} 1: 2,56.10^{9} \text {. }
$$

Les principales bactéries retrouvées chez ce patient sont :

- Fn : la proportion constatée à J0 est de 0,89\% contre $0,62 \%$ à $\mathrm{J} 1$;

- Pg : sa quantité par rapport à la flore totale est de $0,90 \%$ à $\mathrm{J} 0$ et de $0,32 \%$ à $\mathrm{J} 1$;
- $\mathrm{Pm}$ : on retrouve une proportion de $0,44 \%$ à J0 contre $0,19 \%$ à J1 ;

- Le reste des germes retrouvés ont vu leur quantité légèrement augmentée.

\section{Discussion}

Les résultats de cette étude indiquent que l'application de l'ozone au sein des poches parodontales a un réel effet sur la présence bactérienne. Nous constatons une diminution globale de la proportion de toutes les bactéries, sauf exception où certaines bactéries ne diminuent pas mais restent stables.

Certaines bactéries sont plus sensibles que d'autres, mais de façon variable selon les patients.

De façon générale, les bactéries les plus concernées par l'effet bactéricide de l'ozone sont Fusobacterium nucleatum (Fn), Porphyromonas gingivalis $(\mathrm{Pg})$ et Peptostreptococcus micros (Pm).

Fn est souvent isolé dans les formes de parodontites chroniques à évolution lente. II s'agit de l'espèce bactérienne retrouvée en plus grande quantité chez tous les patients. La réduction de sa proportion en dessous de $0,5 \%$ traduit une bonne maîtrise de la flore sousgingivale par le traitement; ce que l'on peut

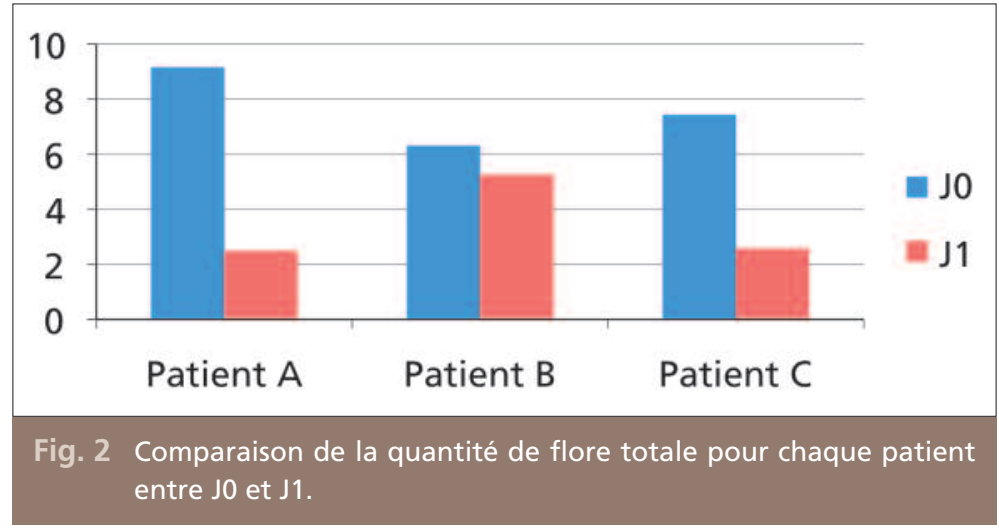


constater chez le patient B. On sait que sa réduction est un bon marqueur de stabilisation de la flore [10]. Quant à Pg, sa présence traduit la phase d'activité de la parodontite chronique. Ce germe a également été retrouvé en grande quantité chez tous les patients de l'étude. L'utilisation de l'ozone a réduit considérablement sa concentration au sein des poches parodontales.

\section{2 e protocole}

Trois patients sont entrés dans ce protocole. Le suivi du patient s'est effectué sur trois semaines soit quatre rendez-vous à une semaine d'intervalle.

- À J0 : quatre sites de prélèvements ont été choisis. Ils sont restés les mêmes pendant toute la durée de l'étude chez un patient donné. Ces prélèvements servent de témoins pour comparer la flore au cours du protocole. Puis une injection à l'ozone est effectuée sur l'ensemble des sites concernés (1re séance).

- À J8 : sonde à ADN au niveau des sites choisis à J0 puis protocole ozone (accompagné toujours du contrôle par sondage parodontal, indices de saignement et d'hygiène). Puis protocole à l'ozone ( $2^{\mathrm{e}}$ séance).
En conclusion, par ce protocole, l'effet bactéricide de l'ozone sur l'ensemble de la flore buccale est clairement démontré, avec des indices d'hygiène inchangés étant donné que le protocole ne s'est étendu que sur deux jours (donc grande fiabilité).

Un second protocole a été mis en place de façon à étendre le traitement à l'ozone sur plusieurs semaines.

- À J16 : sonde à ADN au niveau des sites choisis à $\mathrm{J} 0$ puis protocole ozone. Puis protocole à I'ozone (3e séance).

- À J24 : prélèvements finaux (4e séance).

\section{Résultats}

\section{> Patient 1 (fig. 3 et $\mathbf{4}$ )}

Dans le cas de ce patient, nous remarquons une diminution de la proportion des bactéries testées durant les 3 premières séances puis une augmentation lors de la séance 4 , où nous constatons parallèlement une diminution de la motivation du patient à l'hygiène (augmentation de l'indice de plaque et de saignement).
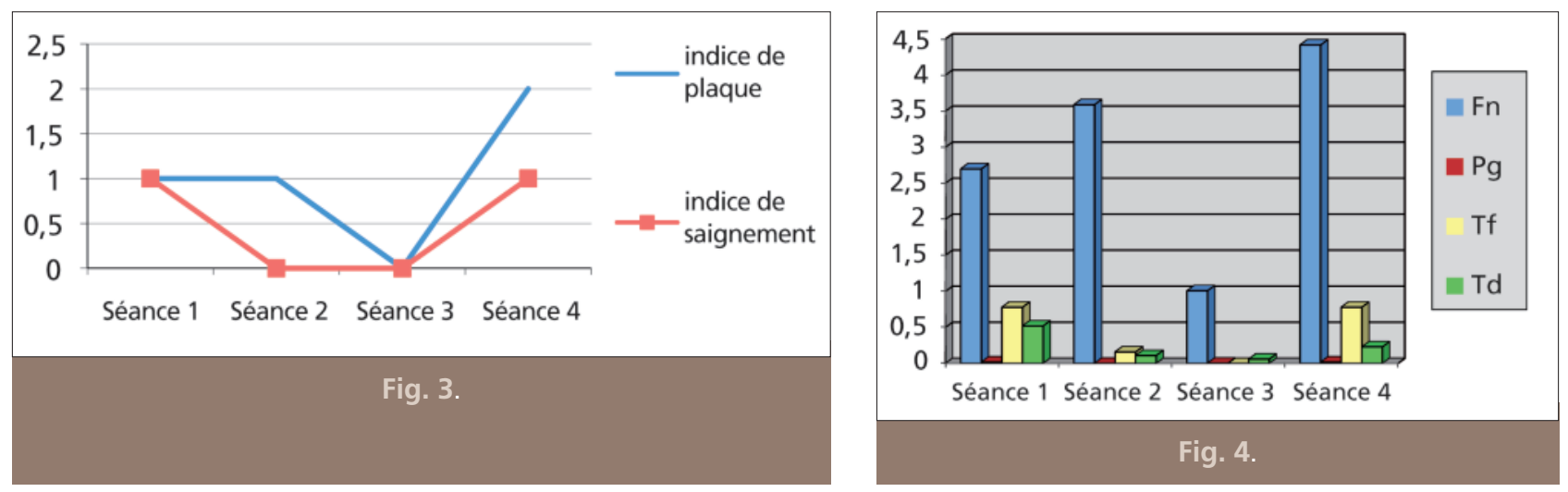

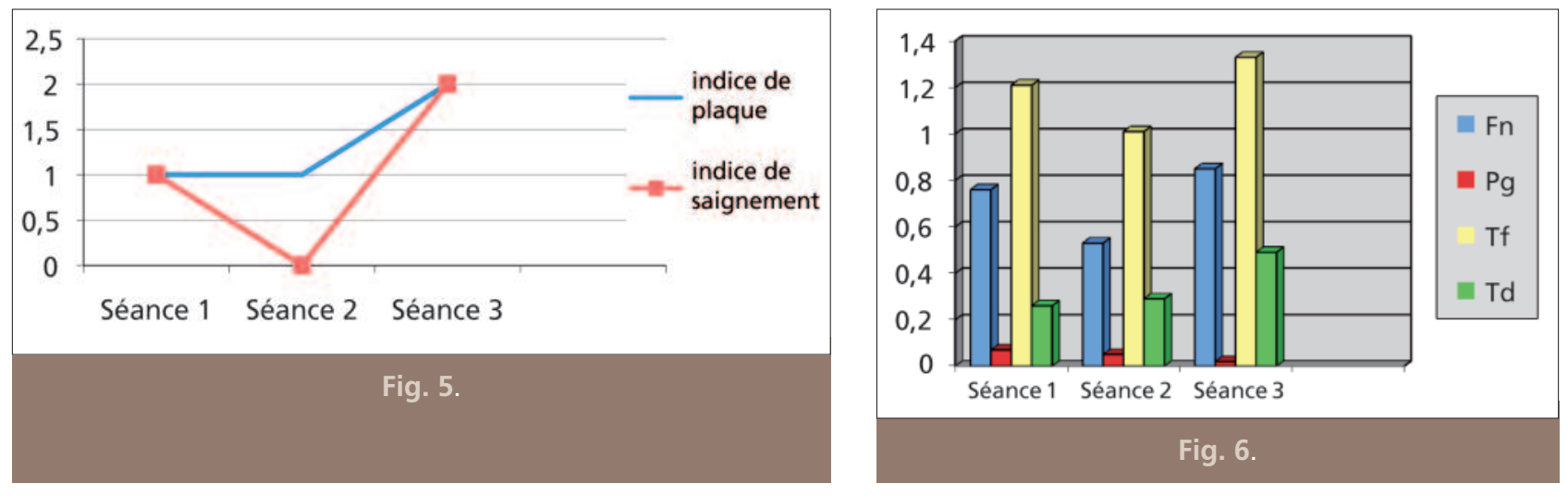

\section{$>$ Patient 2 (fig. $\mathbf{5}$ et $\mathbf{6}$ )}

Dans ce cas, les résultats pour Pg sont positifs, cependant il n'y a pas de réel effet sur les autres bactéries. Ceci s'explique par le fait que, malgré les dires de la patiente, les indices d'hygiène restent élevés. On peut supposer que le patient a eu le temps, entre les séances, de recontaminer les sites traités. Ceci met en exergue un biais dû à la motivation du patient qui vient fausser nos résultats.

\section{> Patient 3 (fig. 7 et 8)}

Chez le sujet 3 , nous constatons une diminution de la proportion de l'ensemble des bactéries:

- Fn passe successivement de 4,99 \% au début de l'étude à $0,69 \%$ à la fin de l'étude ;

- Pg reste stable à $0,03 \%$;
- Tf passe de $0,31 \%$ à $0,18 \%$ à la fin de l'étude ;

- Td passe de $0,6 \%$ à 0,3 \%.

Ces résultats attestent d'une réelle amélioration chez ce patient malgré une démotivation du patient en fin de protocole.

À la suite de cette prise en charge, les patients ont relâché leurs habitudes d'hygiène.

En conclusion, les résultats de cette étude sont concluants également concernant l'effet bactéricide de l'ozone sur les poches parodontales.

L'utilisation de l'ozone semble être une nouvelle piste thérapeutique dans la gestion de I'assainissement d'un environnement parodontal pathogène. Il est à noter que la motivation du patient par les techniques d'hygiène doit être constante pour obtenir une réussite thérapeutique totale.
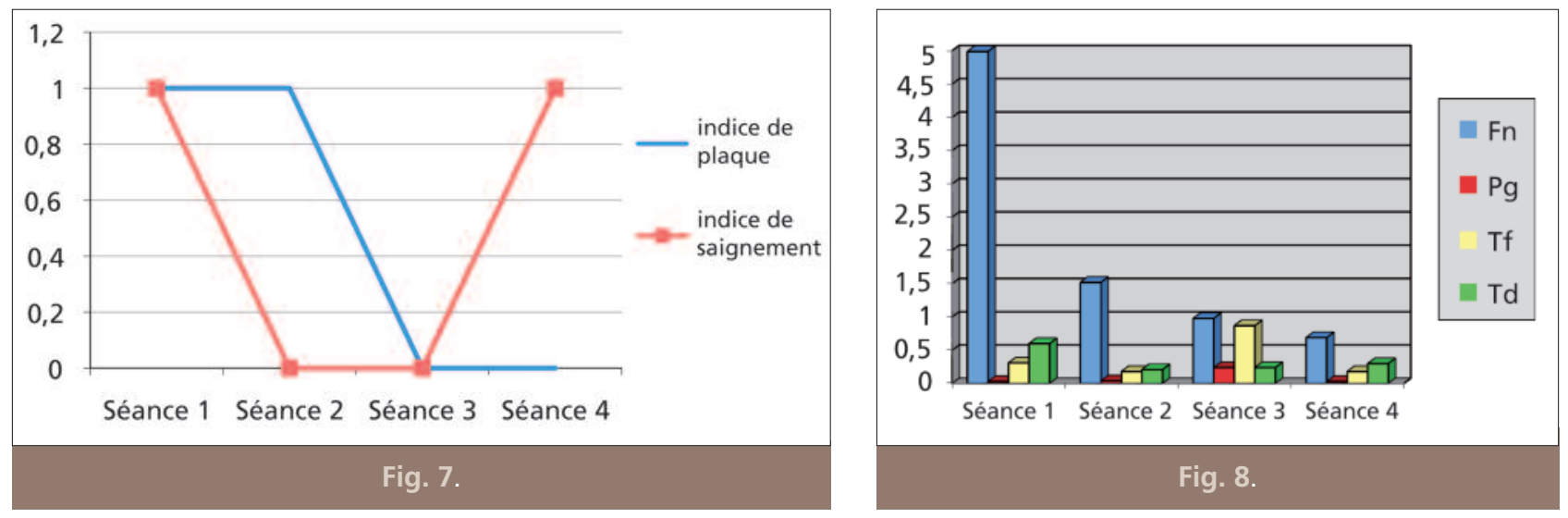


\section{Ozone appliqué sur un cas de péri-implantite}

De par ses propriétés bactéricides sur les bactéries parodontopathogènes, I'utilisation de l'ozone apparaît comme une nouvelle solution thérapeutique dans la gestion des péri-implantites. Pour assainir l'environnement de l'implant, il est nécessaire de ne pas porter atteinte à sa structure : il est indispensable de conserver le col lisse implantaire, mais aussi de ne pas dégrader l'état de surface des spires qui conditionne la régénération tissulaire. L'ozone peut être un bon complément au moyen mécanique qu'est le curetage.

Nous avons donc utilisé l'ozone dans la gestion d'un cas de péri-implantite.

Il s'agit d'une patiente de 65 ans se présentant au centre de soins dentaire de Montpellier pour des récessions vestibulaires au maxillaire sur de nombreuses dents. Après examen clinique, il a été constaté des déhiscences osseuses vestibulaires sur les dents naturelles et particulièrement une poche parodontale de $5 \mathrm{~mm}$ en vestibulaire de l'implant en place de la 24. Le diagnostic de péri-implan-

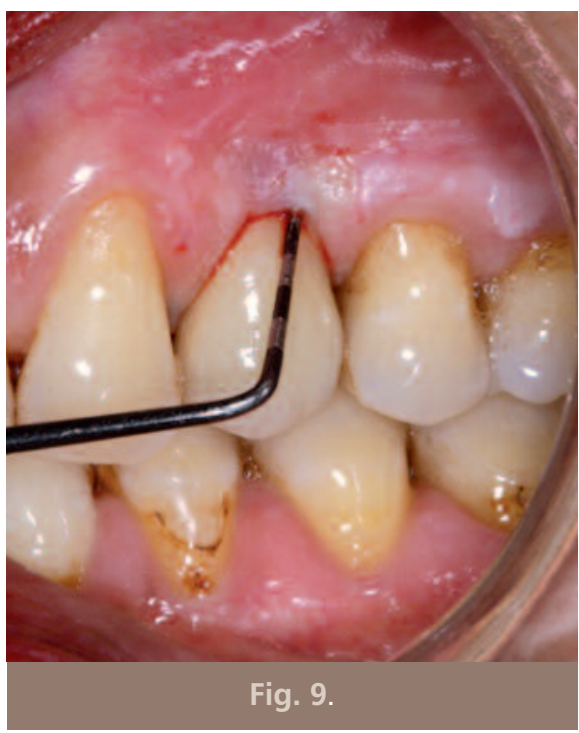

tite d'origine infectieuse a été posé. Nous lui avons proposé un traitement local à l'ozone associé à un comblement osseux et la pose d'une membrane biologique. Ce choix thérapeutique nous est dicté par l'importance de la perte osseuse.

Il est à noter que durant l'intervention, aucun antiseptique ni antibiotique n'a été employé. Seuls des rinçages au sérum physiologique ont été réalisés.

\section{Protocole}

- Sondage parodontal au préalable $(5 \mathrm{~mm})$ / radio rétro-alvéolaire pré-opératoire (fig. 9 et 10).

- Choix des incisions : incision de décharge en distal afin de ne pas aggraver la récession sur la 23 (récession de classe I de Miller de 4 mm) (fig. 11).

- Décollement en demi-épaisseur afin de laisser le périoste en place et de pouvoir glisser la membrane biologique (fig. 12).

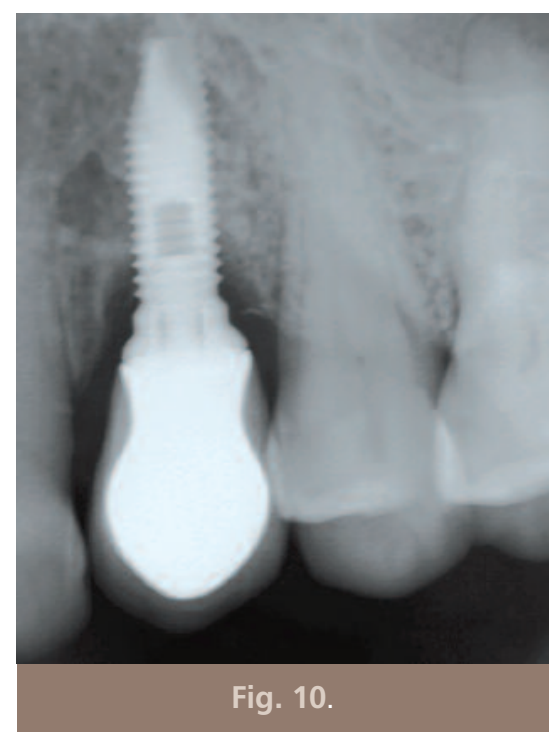


- Curetage du tissu infecté (fig. 13).

- Mise en évidence de la déhiscence et du défaut osseux principalement en vestibulaire (fig. 14).

- Application de l'ozone (à l'aide de l'embout PerioTip) sur la surface de l'implant (fig. 15).

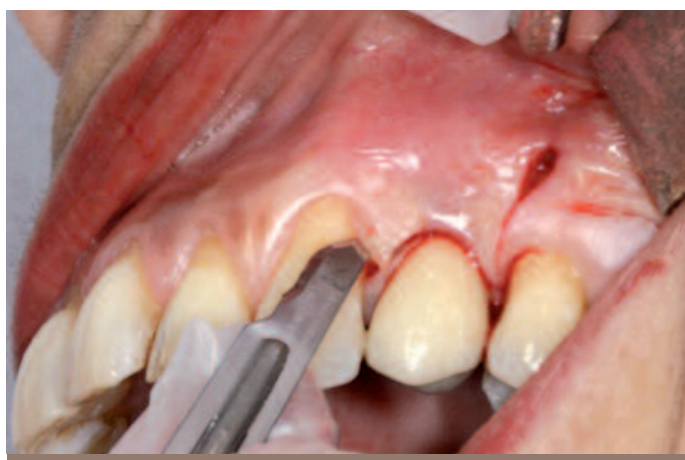

Fig. 11.
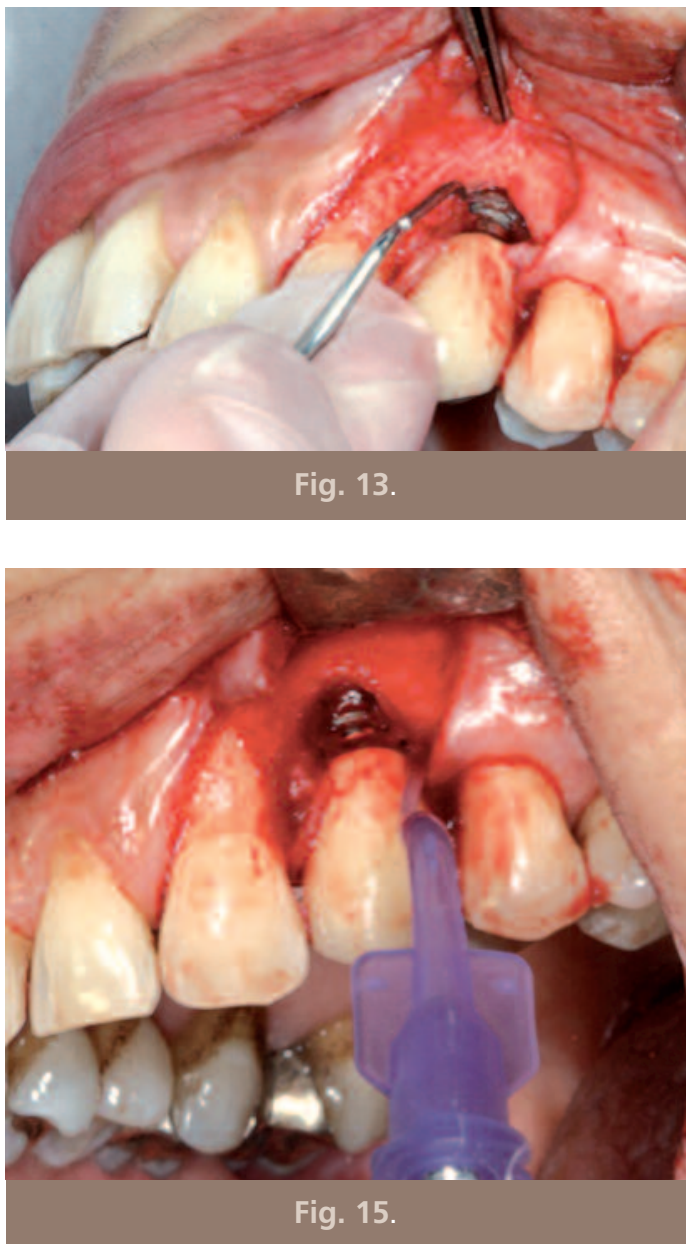

- Comblement osseux associé à une membrane biologique :

- préparation et essayage du patron de la membrane (fig. 16) ;

- comblement au Bioss ${ }^{\circledR}$ et mise en place de la

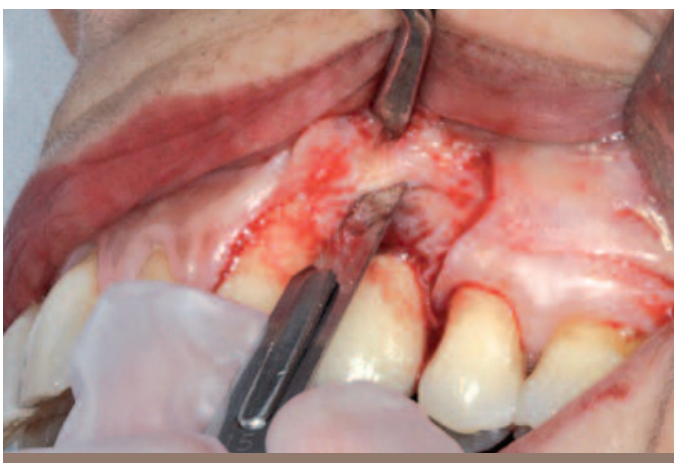

Fig. 12.
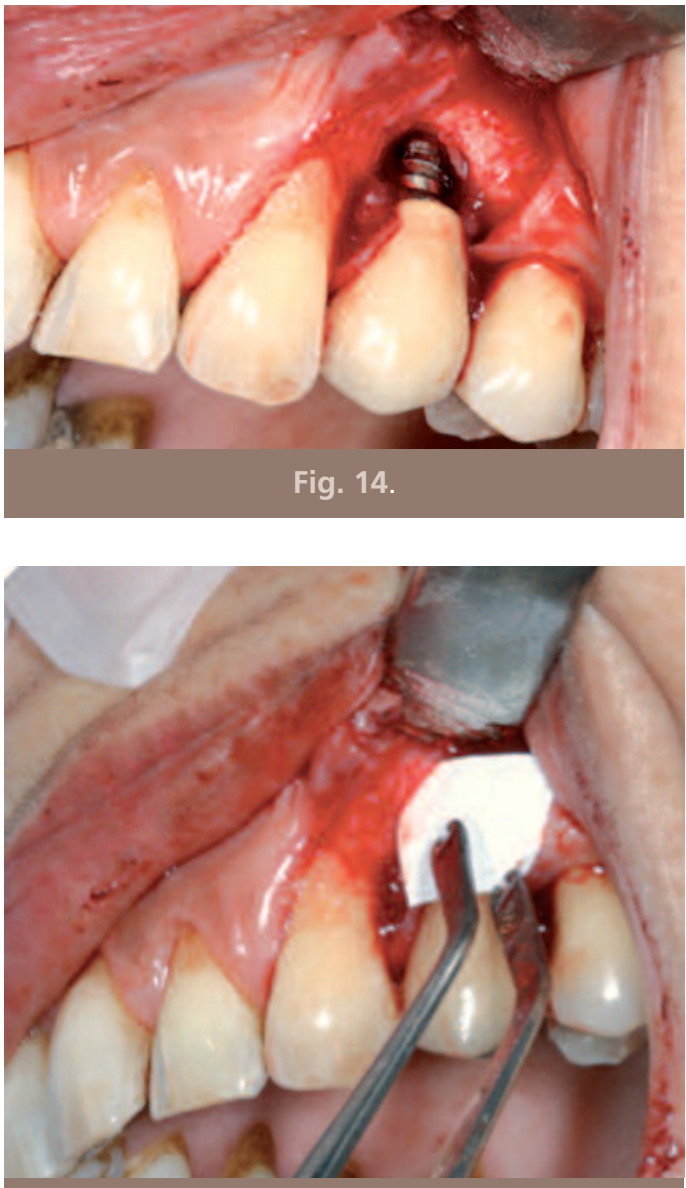

Fig. 16 . 
membrane biologique (Biomend Extend ${ }^{\circledR}$ )

(fig. 17 et 18).

- Sutures (fig. 19 et 20).

- Contrôle à 10 jours (fig. 21).
- Contrôle à 6 mois (ré-intervention en raison d'une greffe sur la 23) (fig. 22 et 23).

On constate une régénération osseuse significative.
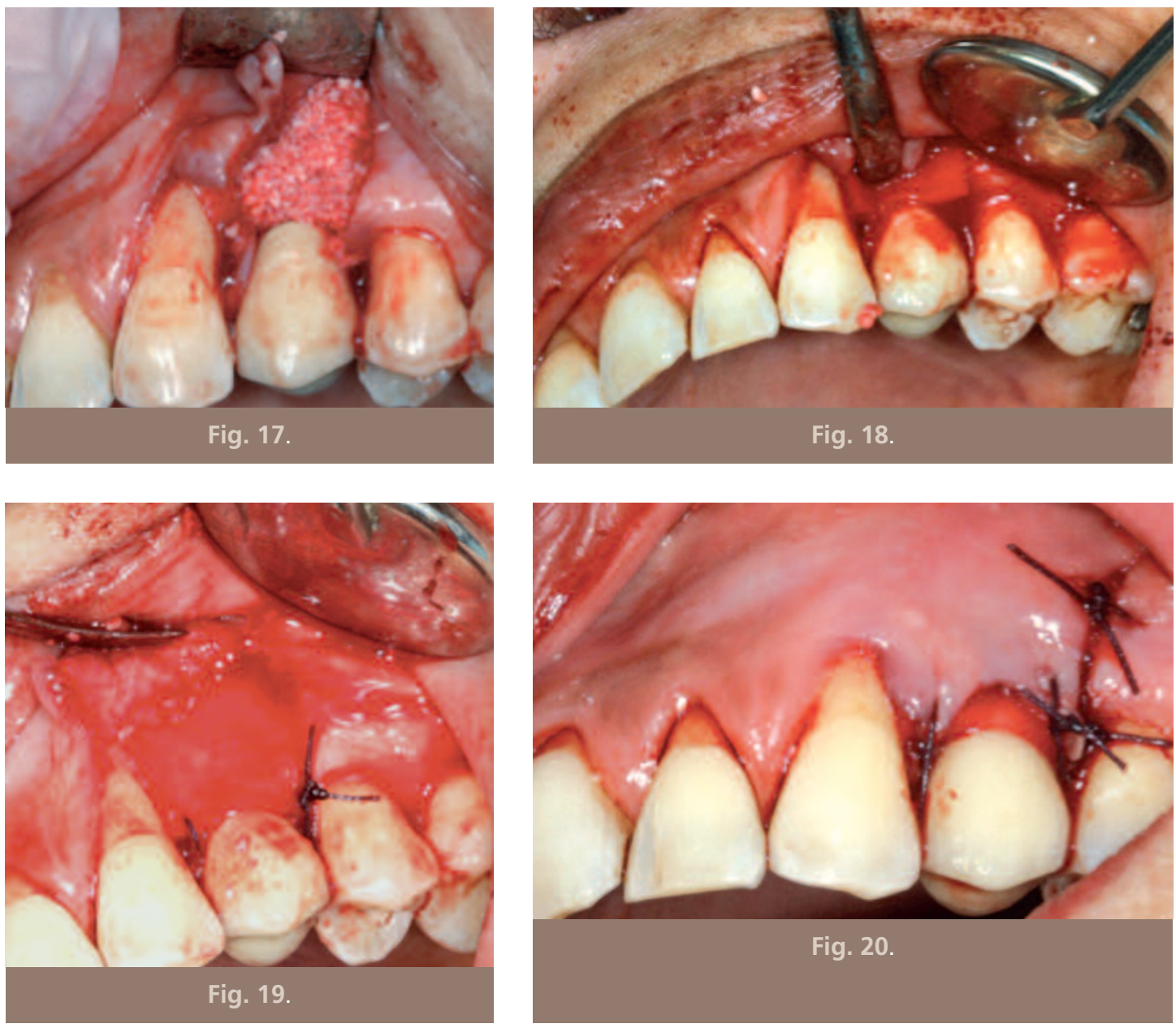

Fig. 20.

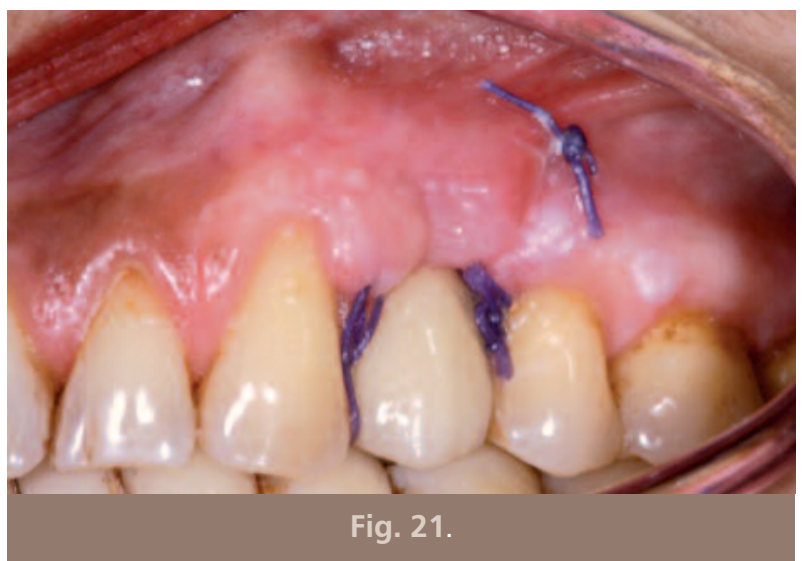


Le caractère infectieux de la maladie a été maîtrisé. Un facteur occlusal a été également contrôlé par la mise en place d'une gouttière de relaxation neuro-musculaire.

Pour conclure, si ce cas n'apporte, bien entendu, pas de solution idéale dans la gestion des péri- implantites, il illustre le fait que de nouvelles pistes thérapeutiques sont explorées ; I'ozone en fait partie.

L'ozone apparaît comme une nouvelle technique efficace qui pourrait compléter notre arsenal thérapeutique déjà existant.
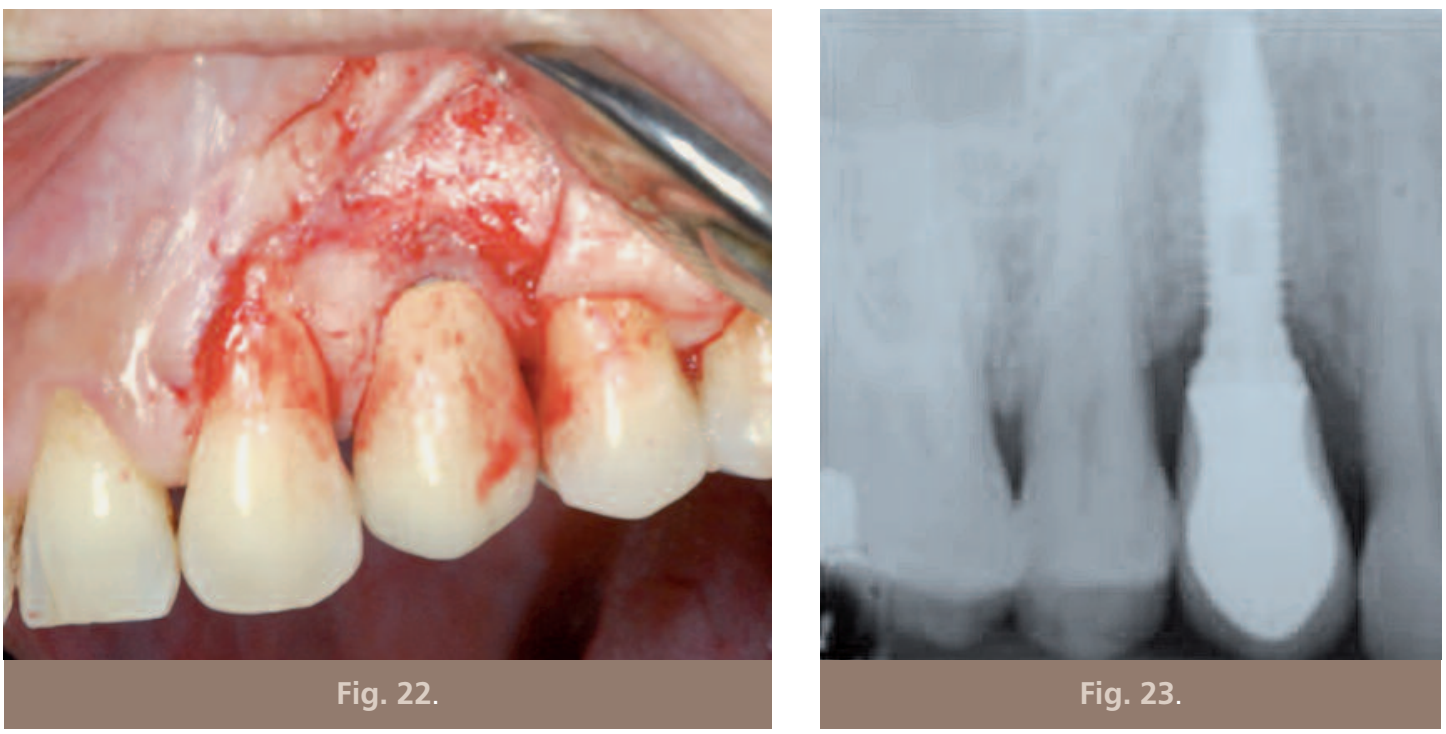

\section{Conclusion}

Les résultats de cette étude sont concluants concernant l'effet bactéricide de l'ozone sur les poches parodontales. L'utilisation de l'ozone semble être un complément dans la gestion de l'assainissement d'un environnement parodontal ou implantaire pathogène, en association avec les techniques habituelles. Nous savons que la recolonisation bactérienne s'effectue dans un délai de trois mois $[4,15]$. Le but serait donc de définir une fréquence de renouvellement du traitement à l'ozone pour obtenir une efficacité thérapeutique totale dans le traitement de la maladie parodontale et péri-implantaire.

\section{Bibliographie}

1. Azarpazhooh A, Limeback $\mathrm{H}$. The application of ozone in dentistry: a systematic review of literature.
J Dent 2008;36(2):104-16.

Epub 2007 Dec 31. 
2. Bain CA.

Smoking and implant

failure-benefits

of a smoking cessation

protocol.

Int J Oral Maxillofac

Implants 1996;11(6):756-9.

3. Brandes $\mathrm{R}$, Beamer B, Holt $\mathrm{S}$,

Kornman K, Lang N.

Clinical-microscopic

observation

of ligature induced

"peri-implantitis"

around osseointegrated

implants.

J Dent Res 1988;67(2):

spec issue, abstr 1397:391-8.

4. Danser MM,

van Winkelhoff AJ,

de Graaf J, Loos BG,

van der Velden $\mathrm{U}$.

Short-term effect

of full-mouth extraction

on periodontal pathogens

colonizing the oral mucous

membranes.

J Clin Periodontol

1994;21:484-9.

5. Harris LG, Mead L, Müller-Oberländer $\mathrm{E}$, Richards RG.

Bacteria and cell cytocompatibility studies on coated medical grade titanium surfaces.

J Biomed Mater Res A 2006;78:50-8.

6. Heitz-Mayfield LJA, Lang NP.

Comparative biology of chronic

and aggressive periodontitis vs. peri-implantitis.

Periodontology 2000

2010;53:167-81.

7. Huth KC, Jakob FM, Saugel B, Cappello C, Paschos E, Hollweck R, Hickel R, Brand K.

\section{Effect of ozone}

on oral cells compared

with established

antimicrobials.

Eur J Oral Sci

2006;114(5):435-40.

8. Lang NP, Brägger U, Walther D, Beamer B, Kornman KS.

Ligature induced peri-implant infectious in the cynomolgsus monkeys.

Clin Oral Implants Res 1993,4(1):2-11.

9. Matarasso S, Quaremba G, Coraggio F, Vaia E, Cafiero C, Lang NP. Maintenance of implants: an in vitro study of titanium implant surface modifications subsequent to the application of different prophylaxis procedures.

Clin Oral Implants Res 1996:7(1):64-72.

10. Mattout $P$, Mattout $C$. Les thérapeutiques parodontales et implantaires.

Paris :

Quintessence International 2003.

11. Mercadier $C$.

La parodontite habituelle de l'adulte : bactériologie et thérapeutique.

Thèse Doct. Montpellier : Univ, 1992.

12. Nagayoshi M, Fukuizumi T, Kitamura C, Yano J, Terashita M, Nishihara T. Efficacy of ozone on survival and permeability of oral microorganisms. Oral Microbiol Immunol 2004:19(4):240-6.
13. Quirynen $M$, Peeters $W$, Naert I, Coucke W, van Steenberghe $D$. Peri-implant health around screw-shaped c.p. titanium machined implants in partially edentulous patients with or without ongoing periodontitis. Clin Oral Implants Res 2001;12(6):589-94.

14. Robert-Maillet C, Missika P. Traitement de la péri-implantite : étude bibliographique. Act Odonto Stomatol 1997; 198:405-23.

15. Serino G, Rosling B, Ramberg P, Socransky SS, Lindhe J.

Initial outcome and long-term effect of surgical and non-surgical treatment of advanced periodontal disease.

J Clin Periodontol 2001;28(10):910-6.

16. Socransky SS, Haffajee AD, Cugini MA, Smith C, Kent RL Jr. Microbial complexes in subgingival plaque. J Clin Periodontol 1998:25(2):134-44.

17. Van Steenberghe $D$,

Naert I, Jacobs R,

Quirynen M.

Influence

of inflammatory reactions vs. occlusal loading on peri-implant marginal bone level.

Adv Dent Res 1999;13:130-5.

18. Vincent JL, Lartigau G. À propos de l'ozone. Rev Fr Odontostomatol 1971;18(7):839-58. 


\section{SUMMARY}

\section{Periodontal diseases and peri-implantitis :} interest of the use of ozone

Julie DUCLOT,

Maxime DELVALLE, Manon SERRE,

Philippe GIBERT

\section{Keywords \\ - periodontitis \\ - peri-implantitis \\ - ozone}

Peridontal and peri-implant diseases have a lot of similarities, in particular their bacterial origin, caracterized by specific bacteria.

After describing the definition of the diseases, we continue with the bacteriology of the disease and their pathogeny mechanism. In this article we also try to conclude on the current anti infections therapies.

Finally, we are going to precise (with a pretiminary clinical study) the use of ozon, gaz which is know and used in medecine (for his bactericidal propertises) since the beginning of the $20^{\text {th }}$ century.

\section{Une pédagogie remarquable, des dessins exceptionnels!}

Véritable outil de perfectionnement post-universitaire, en orthopédie et en orthodontie, cet ouvrage est un travail de praticien et d'enseignant. II s'adresse aux chirurgiens-dentistes qui souhaitent développer leur connaissance de l'orthopédie et de l'orthodontie.

Le livre propose certains modèles théoriques permettant de mieux comprendre l'installation, au cours de la croissance sous le jeu des fonctions, de la délicate architecture crâniomaxillo-faciale, ainsi que la définition, en forme, en volume et en situation dans l'espace, des éléments qui la composent.

Chaque atteinte de cet équilibre organise un désordre occlusal, bien particulier, qu'il faut apprendre à reconnaître.

Cette découverte de la création des dysmorphies précise les cibles étiologiques et conduit à la mise en place d'une stratégie thérapeutique et raisonnée.

La construction d'une occlusion, en accord avec les capacités mécaniques mieux comprises des structures qui portent les arcades, doit assurer un résultat fonctionnel, donc stable.

L'auteur, Jean Flour a créé le CETO en 1991 (Centre d'Enseignement des Techniques Orthodontiques Orthopédiques et Fonctionnelles). II y enseigne la formation avec une pédagogie remarquable et originale issue d'un long travail de synthèse, de recherche et de réflexion. Jean Flour est Docteur en Sciences Odontologiques, Docteur d'Université, Spécialiste Qualifié en Orthopédie Dento-Faciale, et Ancien Maître de Conférence des Universités.

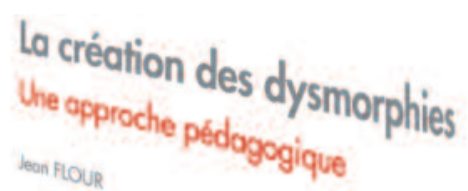

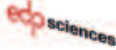

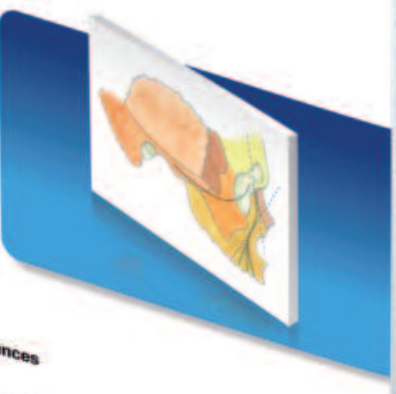

